\title{
Normal Reflection Characteristics of One-Dimensional Unsteady Flow Shock Waves on Rigid Walls from Pulse Discharge in Water
}

\author{
Dong Yan, Jinchang Zhao, and Shaoqing Niu \\ College of Mining Engineering, Taiyuan University of Technology, Taiyuan 030000, China \\ Correspondence should be addressed to Jinchang Zhao; jinchangprofessor@163.com
}

Received 14 October 2016; Revised 19 December 2016; Accepted 19 January 2017; Published 14 February 2017

Academic Editor: Ivo Caliò

Copyright (C) 2017 Dong Yan et al. This is an open access article distributed under the Creative Commons Attribution License, which permits unrestricted use, distribution, and reproduction in any medium, provided the original work is properly cited.

\begin{abstract}
Strong shock waves can be generated by pulse discharge in water, and the characteristics due to the shock wave normal reflection from rigid walls have important significance to many fields, such as industrial production and defense construction. This paper investigates the effects of hydrostatic pressures and perturbation of wave source (i.e., charging voltage) on normal reflection of onedimensional unsteady flow shock waves. Basic properties of the incidence and reflection waves were analyzed theoretically and experimentally to identify the reflection mechanisms and hence the influencing factors and characteristics. The results indicated that increased perturbation (i.e., charging voltage) leads to increased peak pressure and velocity of the reflected shock wave, whereas increased hydrostatic pressure obviously inhibited superposition of the reflection waves close to the rigid wall. The perturbation of wave source influence on the reflected wave was much lower than that on the incident wave, while the hydrostatic pressure obviously affected both incident and reflection waves. The reflection wave from the rigid wall in water exhibited the characteristics of a weak shock wave, and with increased hydrostatic pressure, these weak shock wave characteristics became more obvious.
\end{abstract}

\section{Introduction}

Shock waves are a common physical phenomenon. They are a very thin fluid flow region where the macroscopic state parameters change dramatically. Research on shock waves mainly focuses on propagation and changes to the medium physical states [1]. Shock wave reflection from rigid walls has wide application potential in the fields of oil exploitation, gas treatment, and national defense [2]. High voltage pulse discharge in water is a similar process to an explosion and can generate strong shock waves [3]. There have been many reports regarding shock wave reflection. However, few studies have considered the effect of different hydrostatic pressure on shock wave reflection from a rigid wall. Therefore, this study investigates normal reflection characteristics of shock waves generated by high voltage pulse discharge in water on the rigid wall under different hydrostatic pressures and charging voltage theoretically and experimentally.

\section{One-Dimensional Unsteady Flow Shock Wave Reflection from a Rigid Wall}

2.1. Fundamental Relationship between Incidence and Reflection Waves. Consider a shock wave reflecting from a rigid wall. We define the static medium initial state as 0 with $\rho_{0}$, $p_{0}$, and $u_{0}$; the state behind the incident shock wave $\mathbf{s}_{1}$ as the incidence state 1 with $\rho_{1}, p_{1}, u_{1}$; and the state behind the reflection shock wave $\boldsymbol{s}_{2}$ as the reflection state 2 with $\rho_{2}$, $p_{2}, u_{2}$, where $\rho$ is the density, $p$ is the pressure, and $u$ is the particle velocity. In the reflection process, Figure 1(a) shows typical shock wave reflection curves by time $(t)$ and distance $(x)$, where the rigid wall at $x=0$ is the position of rigid wall, $\mathbf{s}_{1}$ is the incident shock wave, and $s_{2}$ is the reflection shock wave. Figure $1(\mathrm{~b})$ shows pressure $(p-)$ particle velocity $(u)$ curve of shock wave, 0 is the initial state, 1 is the incidence state, 2 is the reflection state, $L_{0}$ is the pressure-particle velocity curve of shock wave under normal condition, and $L_{1}$ is the 


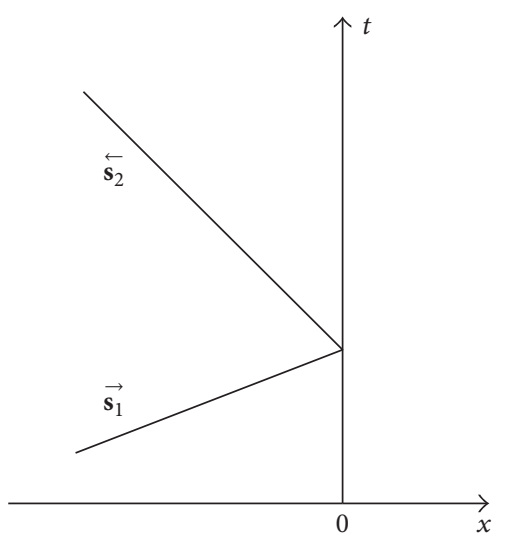

(a)

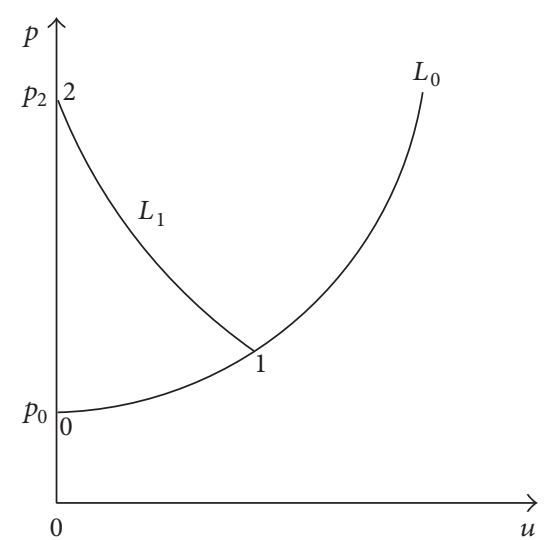

(b)

Figure 1: (a) Transfer time- $(t-)$ distance $(x)$ curve. (b) Pressure- $(p-)$ particle velocity $(u)$ curve of shock wave.

pressure-particle velocity curve of shock wave in reflection process on rigid wall.

Among this, the equations of $p$ - $u$ curves (i.e., $L_{0}$ and $L_{1}$ ) corresponding to shock waves $\mathbf{s}_{1}$ and $\mathbf{s}_{2}$ are [4]

$$
\begin{aligned}
& u=\varphi_{0}(p)=\sqrt{\frac{2}{\rho_{0}}} \frac{p-p_{0}}{\sqrt{(\gamma+1) p+(\gamma-1) p_{0}}}, \\
& u=u_{1}-\varphi_{1}(p)=u_{1}-\sqrt{\frac{2}{\rho_{1}}} \frac{p-p_{1}}{\sqrt{(\gamma+1) p+(\gamma-1) p_{1}}},
\end{aligned}
$$

respectively, where $\gamma$ is the medium parameter, which will be mentioned in detail below. The wall is rigid, that is, fixed, so the particle velocity behind the reflection wave $u_{2}=0$, and the intersection of curve $L_{1}$ with the $p$ axis (Figure 1(b)) is the pressure behind the reflection wave, that is, $p_{2}$. For that case, from (2),

$$
u_{1}=\sqrt{\frac{2}{\rho_{1}}} \frac{p_{2}-p_{1}}{\sqrt{(\gamma+1) p_{2}+(\gamma-1) p_{1}}} .
$$

The incidence of state 1 is the intersection of curve $L_{0}$ with curve $L_{1}$. Substituting state $1\left(p_{1}, u_{1}\right)$ into $(1)$, then

$$
u_{1}=\sqrt{\frac{2}{\rho_{0}}} \frac{p_{1}-p_{0}}{\sqrt{(\gamma+1) p_{1}+(\gamma-1) p_{0}}}
$$

at the intersection, and solving (3) and (4) and considering the Rankine-Hugoniot relations,

$$
\frac{p_{2}}{p_{1}}=\frac{(3 \gamma-1) p_{1} /(\gamma+1) p_{0}-(\gamma-1) /(\gamma+1)}{(\gamma-1) p_{1} /(\gamma+1) p_{0}+1}
$$

Thus, the main parameter effecting the pressure relationship between the incidence and reflected wave is $\gamma$, which is not constant for different media conditions.
2.2. Solution for the Medium Parameter $\gamma$. The mass, momentum, and energy of the shock wave on the discontinuity surface follow conservation relations, which can be expressed for a one-dimensional plane wave as [5]

$$
\begin{gathered}
\rho(D-u)=\rho_{0}\left(D-u_{0}\right), \\
\rho(D-u)^{2}+p=\rho_{0}\left(D-u_{0}\right)^{2}+p_{0}, \\
e+\frac{p}{\rho}+\frac{1}{2}(D-u)^{2}=e_{0}+\frac{p_{0}}{\rho_{0}}+\frac{1}{2}\left(D-u_{0}\right)^{2},
\end{gathered}
$$

where $D$ is the shock wave velocity and $e$ is the internal energy of medium. Water is a kind of special condensed medium, and the initial hydrostatic pressure $\left(p_{0}\right)$ can be exerted. Moreover, water has typical hydromechanical characteristics, which are similar to gases. For a shock wave in water, the practical state equation can be expressed as [6]

$$
p=c_{0}^{2}\left(\rho-\rho_{0}\right)+(\gamma-1) \rho \cdot e,
$$

where $c_{0}$ is the sound velocity at the initial water pressure. The pressure behind the shock wave is much higher than the hydrostatic pressure in the initial state, and the initial pressure can be ignored. Therefore, the medium of shock wave front is in the initial state, it can be considered that $e_{0}=0$, and since the fluid is static in the initial state, $u_{0}=0$.

Considering the Prandtl equation, $\left(D-u_{0}\right)(D-u)=c_{*}^{2}$, where $c_{*}$ is the velocity when $|D-u|=c$, that is, critical velocity, we can express (6) and (7) as [6]

$$
u=\frac{2}{\gamma+1}\left(D-\frac{c_{0}^{2}}{D}\right)
$$

Los Alamos National Laboratory has shown a linear relationship between shock wave velocity $(D)$ and particle velocity behind the wave [7]; that is,

$$
D=c_{0}+\lambda u,
$$

where $\lambda=1.0502$ for water. Therefore, substituting (8) into (9),

$$
\gamma=2 \lambda\left(1+\frac{c_{0}}{D}\right)-1 .
$$


If (10) is substituted into (5), the pressure of the reflection shock wave $\left(p_{2}\right)$ can be expressed by $p_{0}, p_{1}, c_{0}$, and $D$. Therefore, reflection experiments of shock waves on rigid walls should be focused on the pressures and velocities of the incidence and reflection waves.

\section{Experimental}

3.1. Fundamental Process. During the breakdown of water by high voltage pulse discharge, very high energy concentration is released, causing high temperatures and high pressure plasma [8]. The pressure inside the plasma is much higher than water pressure at the boundary, and the plasma expands outward rapidly, compressing the water and forming the shock wave [9].

We first consider the angle between the shock wavefront and the rigid wall. If the planar wavefront forms a certain intersection angle with the rigid wall, the shock wave reflection will show the regular reflection or Mach reflection [10], which has a certain interference on the research of the normal reflection of shock wave; as a result, the experimental data acquisition will also be interfered. Therefore, the parallel between the planar wavefront and the rigid wall is the ideal condition for the research on the normal reflection of shock wave.

Experiments were performed in a purpose built shock wave tube and the evolution of the shock wavefront along the tube analyzed. As it propagates, the shock wave generates frictional resistance and viscous drag with the medium, heat transfer, and heat radiation. With increasing propagation distance, the boundary condition of a rigid wall means the energy of the shock front gradually decreases [11]. This process may be divided into four stages:

(1) Initial stage. The typical spherical wave is formed by the point source discharge.

(2) Cylindrical wave stage. When the incidence angle formed between the spherical wave and the tube wall is small, regular reflection will occur. With increased propagation distance, incidence angle increases, and the spherical wave gradually becomes cylindrical wave.

(3) Mach stem stage. When the incidence angle between the cylindrical wave and tube wall exceeds some critical value, Mach reflection occurs. The reflection wave does not directly contact the tube wall, rather the Mach stem distributes around the cylindrical wave and vertically contacts the tube wall.

(4) Plane wave stage. With increasing incidence angle of cylindrical wave in the propagation process, the length of Mach stem increases, and the angle of the reflection wave also increases because it is impossible to be strengthened in any radial direction of a wavefront due to the Mach stem. When the reflection angle exceeds a critical value, the reflection wave gradually overlaps on the wavefront and develops into a plane wave with uniform energy distribution [12].

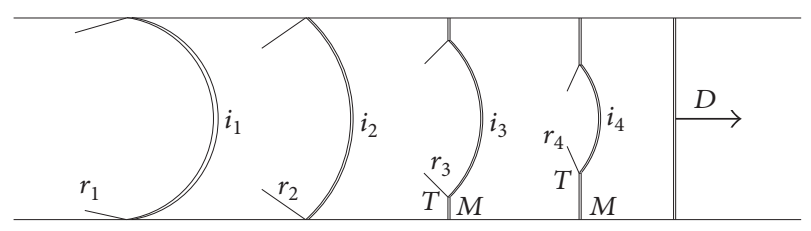

FIGURE 2: Variation trend of shock wavefront in the propagation process along the tube.

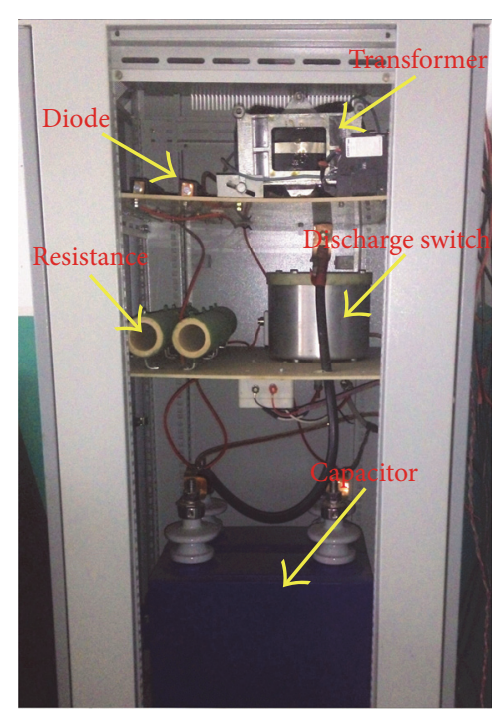

FIGURE 3: Actual high voltage pulse power supply.

Smaller inner tube diameter produces shorter shock waves forming the Mach stem and hence shorter formation time of the planar wave. In this experiment, the propagation distance to the rigid wall was set to be much larger than the tube inner diameter, which was very small. Therefore, shock wave propagation close to the rigid wall was planar wave. Figure 2 demonstrates the shock wave characteristics for this case, where $i_{1}, i_{2}, i_{3}$, and $i_{4}$ are incidence shock waves; and $r_{1}, r_{2}$, $r_{3}$, and $r_{4}$ are the corresponding reflection waves; $M$ is the Mach stem; $T$ is the triple-point of the Mach stem; and $D$ is the velocity of planar wave.

3.2. Equipment. The equipment comprised the tube discussed above and a system for measuring pulse discharges in water. Figure 3 shows the high voltage pulse generating equipment, which provided $6-15 \mathrm{kV}$ DC high voltage with rated capacitance $60 \mu \mathrm{F}$ and energy storage (maximum) $7 \mathrm{~kJ}$.

Figure 4 shows a diagram and photograph of the electrode. The electrodes were constructed from coaxial steel tube and copper bar. The space between the positive and negative electrodes was $5 \mathrm{~mm}$. The voltage threshold can be set manually and discharge of the electrodes in water was conducted using a transmission cable and then recorded by the test system [13].

Figure 5 shows the shock wave test platform for high voltage discharge in water. The main instrument was constructed from high strength carbon steel. Inner and outer diameters 

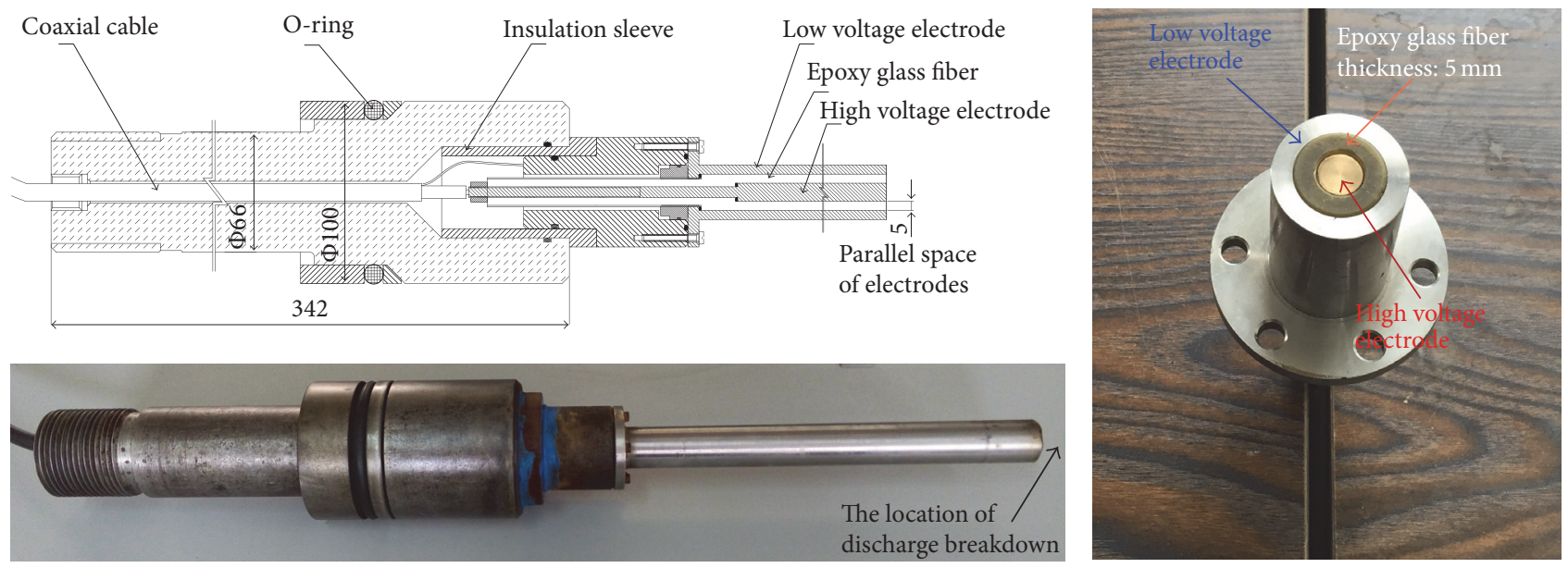

FIGURE 4: Picture of the real electrode and its structural diagram.

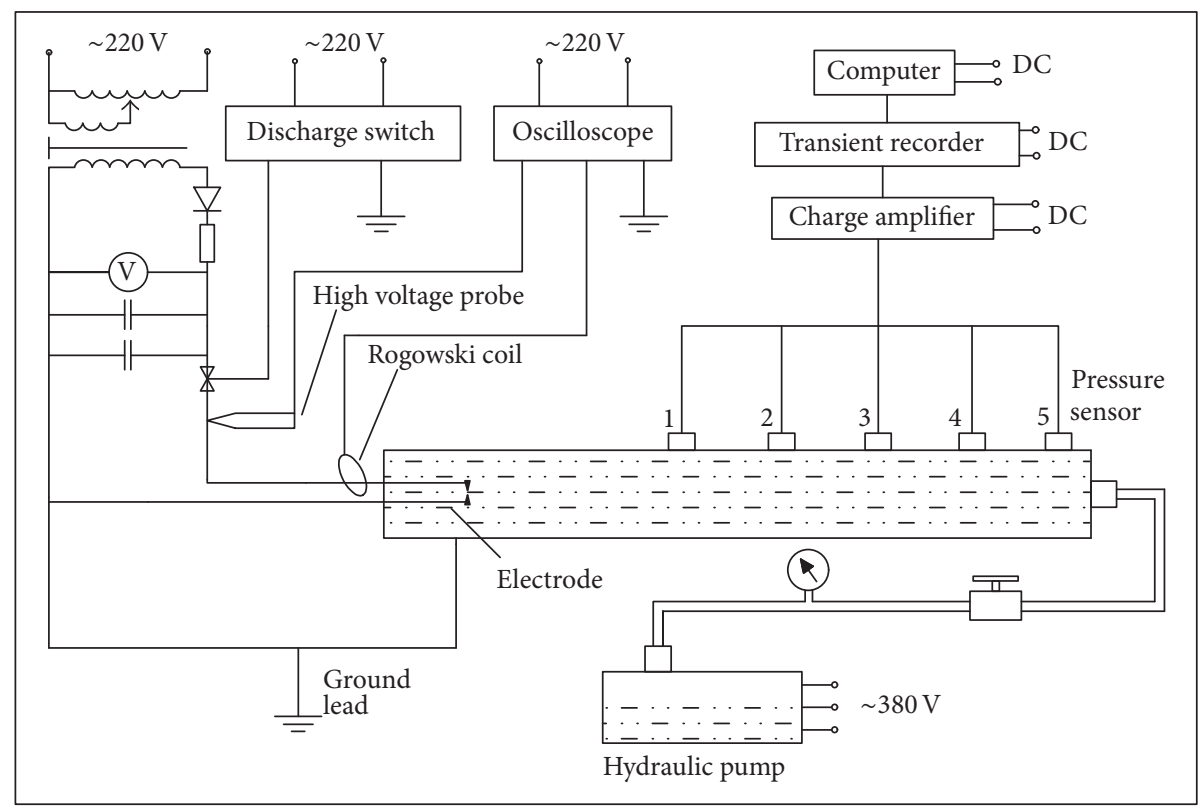

FIGURE 5: Experimental platform (the first part of the experiment).

and tube lengths were 100, 135, and $4000 \mathrm{~mm}$, respectively. Importantly, the length of tube was much larger than the inner diameter. The electrodes were located in the center of the tube, parallel to the tube axis. Hydrostatic pressure in the tube was adjusted using a pressure pump to provide preset environments. Five pressure sensors were uniformly distributed on one side of the tube wall, $0.5 \mathrm{~m}$ apart, with the distance from the electrode to the nearest sensor connector (sensor 1) being $1 \mathrm{~m}$. The tube was filled with tap water with conductivity approximately $1.2 \mathrm{~S} / \mathrm{m}$ and temperature approximately $10^{\circ} \mathrm{C}$. The sensors transmitted signals to a data acquisition recorder and the shock wave pressure and velocity extracted.

The strong DC voltage caused electrode discharge, and so the test system requirements for frequency response and interference resistance were high. The test system comprised a Rogowski coil, P6015A high voltage probe, DSO6014A oscilloscope, CY-214 pressure sensors, YE5853 charge amplifier, transient recorder, and computer.

Sensitivity of the Rogowski coil was $38 \mathrm{kA} / \mathrm{V}$, which provided sufficient precision to measure the pulse current [14]. The P6015A high voltage probe also provided sufficient acquisition accuracy for the transient voltage [15], with maximum input voltage $20 \mathrm{kV}$, bandwidth $75 \mathrm{MHz}$, and rise time $4.0 \mathrm{~ns}$. Sampling rate of the DSO6014A oscilloscope was set at $4 \mathrm{GSa} / \mathrm{a}$, and the maximum measurement range of the piezoelectric pressure sensors was $200 \mathrm{MPa}$ and the nonlinear error of pressure sensor is less than 1\%. The YE853 charge amplifier had bandwidth of $2 \mu \mathrm{Hz}-100 \mathrm{kHz}$, and the YE6231 signal recorder was set with sampling frequency $96 \mathrm{kHz}$. 


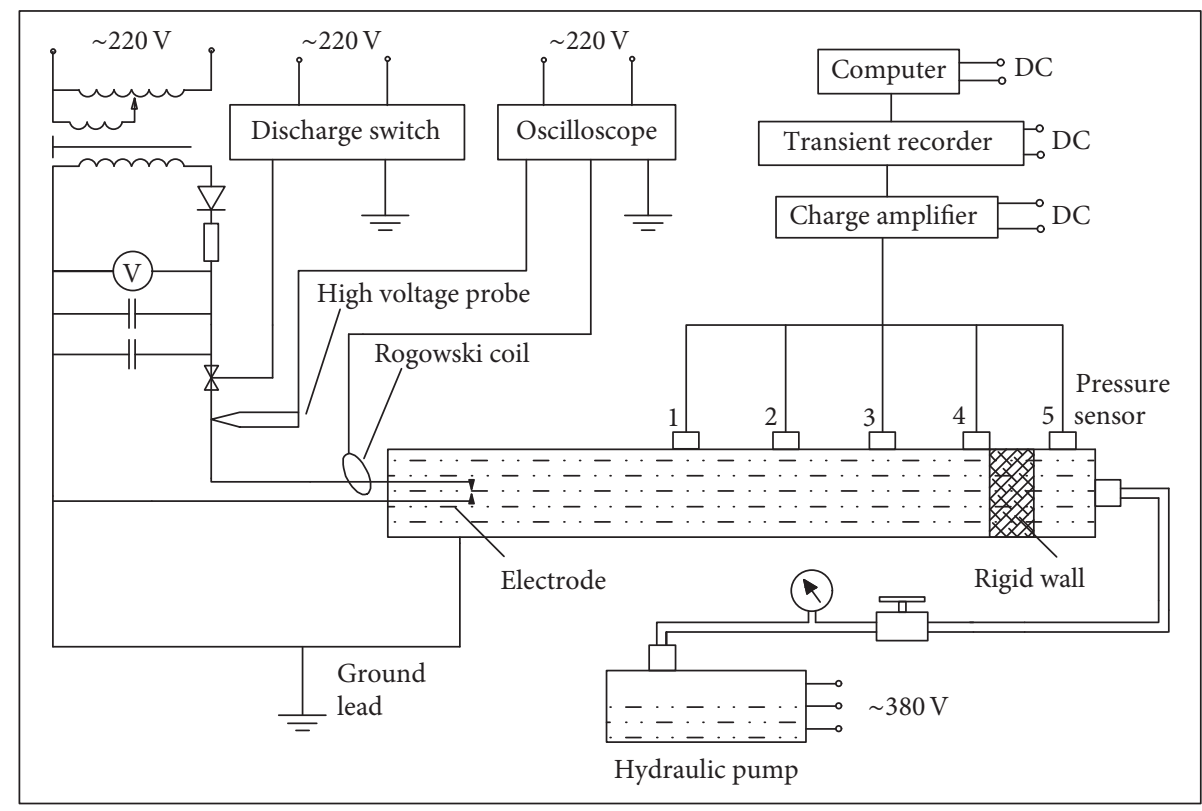

FIGURE 6: Experimental platform with rigid wall added (the second part of the experiment).

Thus, the test system provided significant advantages, such as simple corollary equipment, high sampling frequency, and good repeatability.

3.3. Experimental Scheme. The experiments focused on the reflection of shock waves at the rigid wall for peak pressures and wave velocity under different hydrostatic pressures and charging voltages. Experimental variables included charging voltage and hydrostatic pressure. Charging voltage was set at $8,9,10,11,13$, and $15 \mathrm{kV}$, and the hydrostatic pressure was set at $0,1,2,3,4,6$, and $8 \mathrm{MPa}$. Each group of independent experiments consisted of a different charging voltage and hydrostatic pressure. Hydrostatic pressure of $0 \mathrm{MPa}$ did not mean true zero pressure. To guarantee the tube was filled with water, an external water pressure of approximately $0.02 \mathrm{MPa}$ was applied. Sensor 4 was used as the point to investigate the shock wave reflection on the rigid wall, and the other sensors were used as the auxiliary measurement points to obtain the shock wave velocity.

The experiments were divided into two parts. In the first part, the peak pressure and incidence wave velocity at sensor 4 were measured directly using the device in Figure 5. In the second part, the peak pressure and approximate velocity of the reflection shock wave at sensor 4 were measured. For the second part, a rigid wall was added, as shown in Figure 6, to provide normal reflection of the shock wave. Sensor 4 was close to the rigid wall, to ensure the peak pressure of the reflection shock wave could be measured. In the second part, sensor 5 was behind the rigid wall, so only reflection wave data collected by the first four sensors were considered.

The two parts of the experiments were conducted independently, but we ensured all parameters, including charging voltage and hydrostatic pressure, were consistent. A group of complete experiments could be constituted by combining the experimental data in two parts. Therefore, peak pressures of the incidence and reflection waves could be accurately obtained for each group of experiments and the incidence and reflection wave velocities calculated from the slope of the time-distance curve.

3.4. Measurement of High Voltage Discharge. High voltage discharge in water is similar to an explosion and the discharge energy is instantaneously injected into the plasma channel in an extremely brief period. The pulse discharge can be expressed as [16]

$$
L \frac{d i}{d t}+R(t) i+\left(\frac{1}{C}\right) \int i d t=0
$$

where $L$ is the lead inductance, a very small constant; and $C$ is the capacitance, in this study $C=60 \mu \mathrm{F}$; and the equivalent resistance $R(t)$ is preliminarily determined by initial voltage and liquid conductivity and can be expressed as $R(t)=$ $R_{c}+R_{w}$, where $R_{c}$ is the pure resistance of the circuit and is very small, and $R_{w}$ is the equivalent resistance between the electrodes, which can be expressed by a time-varying second-order homogeneous differential equation. Electrodes spacing was $5 \mathrm{~mm}$, and the equivalent resistance can be determined from the initial voltage and conductivity of the liquid. An accurate numerical solution could be obtained, and the calculation results were all within $0.2 \Omega$ [17].

To ensure the discharge processes in both parts of the experiments were consistent, the discharge process was accurately measured to reduce errors. The discharge measurement system comprised a Rogowski coil, high voltage probe, and oscilloscope. Figure 7 shows the time history curve of the discharge voltage, $U(t)$. The peak $\left(U_{m}\right)$ is the charging voltage, which was an important parameter for the experiment. Figure 8 shows the time history curve of discharge current, $I(t)$. The fluctuation of voltage and current could objectively 


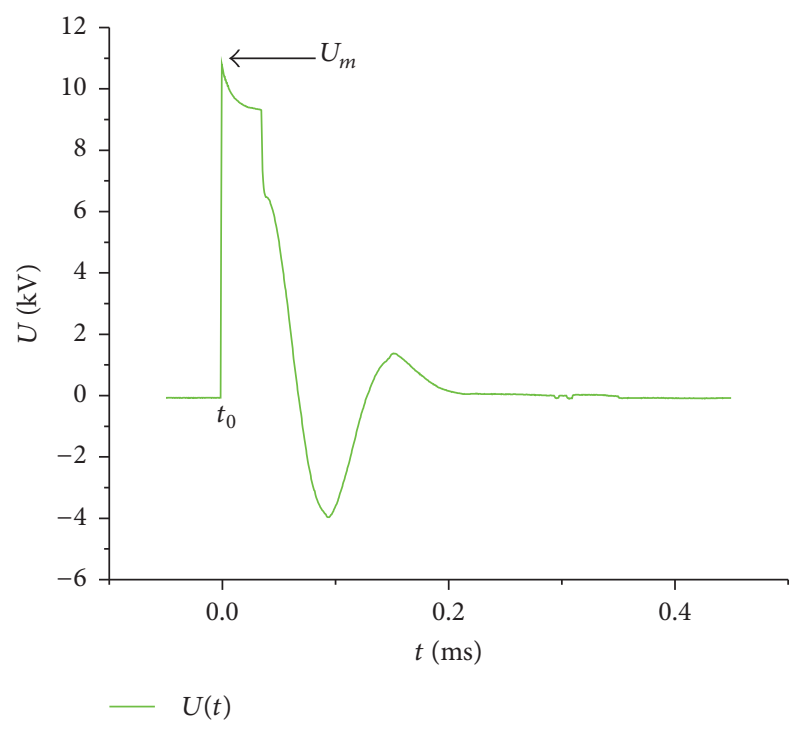

FIgURE 7: Time history curve of the discharge voltage $U(t)$.

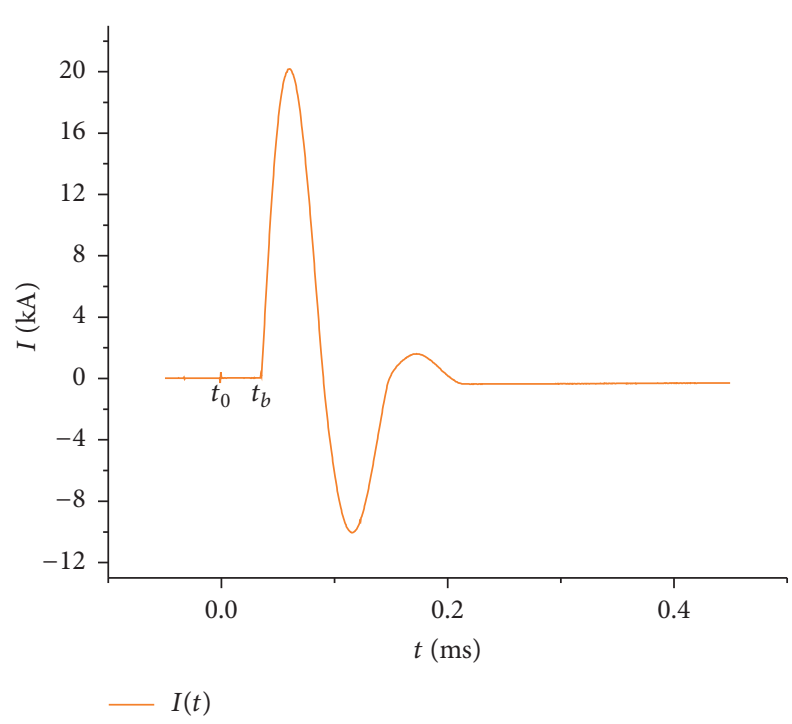

FIGURE 8: Time history curve of the discharge current $I(t)$.

reflect the discharge effect by combining with the differential equation of $R L C$ loop.

In Figures 7 and $8, t_{0}$ and $t_{b}$ are the moments of discharge and breakdown, respectively. The period from $t_{0}$ to $t_{b}$ is the time to form the plasma channel, and $t_{b}$ is the cut-through moment of the plasma channel, that is, when the water is struck through to form the discharge loop.

The experiments focused on normal reflection of the shock wave, so the characteristics of the pulse discharge are not discussed here [18]. However, we were careful to ensure all charging voltages corresponding to one group of complete experiments were highly consistent, including the current waveforms. Thus, each electrical discharge within the group was as much as possible uniform, thereby increasing experimental precision.

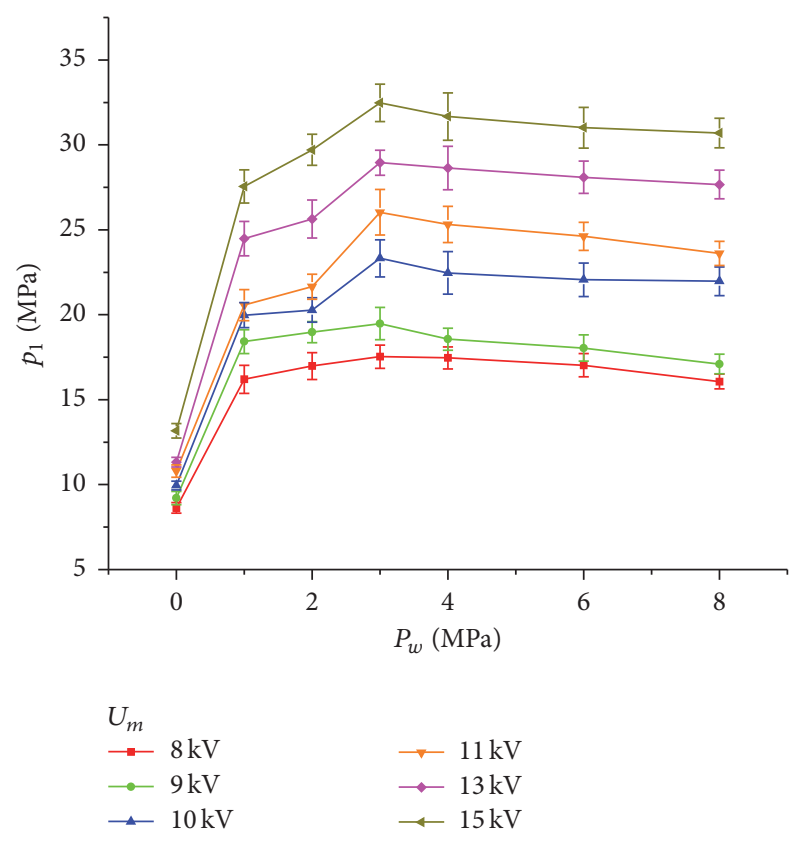

FIGURE 9: Peak pressure of the incident shock wave at sensor 4, $2.5 \mathrm{~m}$ from the electrode.

\section{Normal Reflection of the Shock Wave from the Rigid Wall}

4.1. Characteristics of the Incident Wave. The high voltage pulse discharge instantaneously injects the energy into the plasma channel. The surrounding water is strongly compressed, producing sudden increases in pressure, density, and velocity, and forming the initial shock wave.

The characteristics of the incident shock wave were the peak pressure $\left(p_{1}\right)$ and velocity $\left(D_{1}\right)$, measured at sensor 4 , $2.5 \mathrm{~m}$ from the electrode. The first part of the experiment (as shown in Figure 5) defined the relationships between charging voltage $\left(U_{m}\right)$, hydrostatic pressure $\left(P_{w}\right)$, peak pressure $\left(p_{1}\right)$, and velocity $\left(D_{1}\right)$ of the incidence wave. For each charging voltage and hydrostatic pressure, the peak pressure of the incident shock wave was measured at sensor 4 , as shown in Figure 9.

Peak pressure increases with increasing $U_{m}$ for fixed $P_{w}$. Increasing $U_{m}$ not only increases the energy injected into the plasma channel, but also reduces energy leakage in the breakdown process, with both effects increasing $p_{1}$ [17].

For fixed charging voltage is fixed, the $p_{1}$ at $P_{w}=0 \mathrm{MPa}$ is much lower than for $P_{w}=1-8 \mathrm{MPa}$, which illustrates the influence of hydrostatic pressure on shock wave transfer. As $P_{w}$ increases, voids in the water, such as small bubbles, reduce, density increases, and shock wave attenuation weakens. Therefore, attenuation of the shock wave at $P_{w}=$ $0 \mathrm{MPa}$ is much higher, and increased hydrostatic pressure can promote shock wave transfer. The transfer distance is between electrode and sensor 4.

As $P_{w}$ increases from $0-8 \mathrm{MPa}, p_{1}$ first increases $(0-$ $3 \mathrm{MPa}$ ) then decreases (3-8 MPa). This is mainly due to the influence of small bubbles and hydrostatic pressure on the discharge breakdown. 
First, due to the existence of the original small bubbles in tap water, the breakdown field strength of water medium is lower, resulting in the easy breakdown of water medium by the electrical discharge. In other words, the small bubbles are advantageous to the cut-through of plasma channel in discharge breakdown process of water medium, promoting the breakdown [19]. The breakdown mechanism of high voltage discharge in water is explained: Kao [20], Yanshin et al. [21], and Ushakov [22] proposed the classical theory "breakdown gasification water" by many experiments. The electrolysis and ionization breakdown phenomena will occur when there is a high potential difference between electrodes in water. Firstly, the electrolysis phenomenon occurs, which can be described as below: the water is gasified by electrolytic heating, and the generated small bubbles are adhered onto the electrode surface. Secondly, the ionization breakdown phenomenon occurs. A channel with very strong ray appears between electrodes followed by rapid expansion process that is similar to the explosive detonation.

The whole "breakdown gasification water" process can be described as below: the partial temperature of water rises rapidly due to the Joule heating effect of ionic current in electrode. The water is gasified to small bubbles at high temperature. The gas molecules in bubbles are ionized at high temperature and high voltage. The ionization process occurs in bubbles to form a conduction region. The conductivity in conduction region is nice. Therefore, the discharge breakdown can be observed through large pulse current.

There are some small gas bubbles in tap water, and they can be adhered onto the electrode. We call these gas bubbles original small bubbles (in order to distinguish the small bubbles generated in gasification by discharge heating). The presence of original bubbles weakens the gasification process by discharge heating. The gases in original bubbles can be directly used for ionization, realizing the breakdown.

When the external field strength is fixed, the presence of original bubbles will greatly reduce the energy required for gasification by discharge heating. The saved energy can participate in the ionization breakdown process. As a result, the breakdown energy in plasma channel increases, and the corresponding peak pressure of shock wave also increases (the energy in heating gasification process is meaningless to the formation of shock wave). In other words, the heating gasification field strength and ionization breakdown field strength are required for the water medium without original bubbles in discharge breakdown process, while only ionization breakdown process is required for the water medium with original bubbles. Therefore, the breakdown field strength of water medium with original bubbles is much lower than that of water medium without original bubbles.

The volume of original bubbles will decrease according to " $p V=n R T$ " when the hydrostatic pressure increases. However, the original bubbles can also effectively participate in the ionization breakdown process when $P_{w}$ is $0-3 \mathrm{MPa}$. When the hydrostatic pressure increases to a certain value (the certain value should be in $3 \mathrm{MPa}-4 \mathrm{MPa}$ ), the volume of these original bubbles is too small to balance the external high hydrostatic pressure, much original bubbles breaking and scattering, and cannot participate in the ionization breakdown.

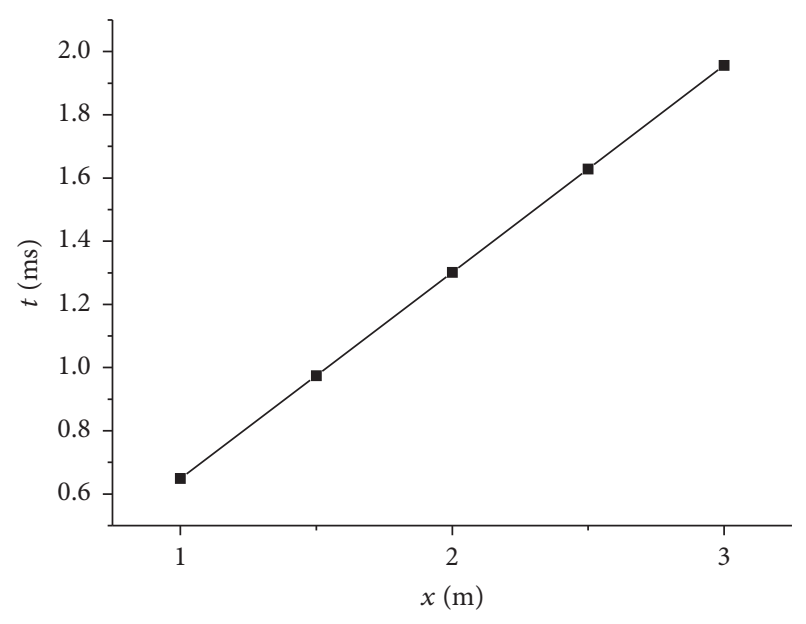

FIgURE 10: Transfer time- $(t-)$ distance $(x)$ curve for the incident shock wave when $P_{w}=1 \mathrm{MPa}$ and $U_{m}=9 \mathrm{kV}$.

Second, the increase of hydrostatic pressure hinders the formation of plasma channel and increases the breakdown resistance accordingly, leading to the decrease of energy injected into the plasma channel. Therefore, increasing $P_{w}$ had an inhibitory effect on the formation of shock wave.

When $P_{w}$ is $0-3 \mathrm{MPa}$, the discharge breakdown is not obviously inhibited by the hydrostatic pressure due to the presence of original small bubbles in water medium. Also, increased $P_{w}$ promotes shock wave transfer. Therefore, the promotion effect of $P_{w}$ on shock wave transfer at this stage is larger than the inhibitory effect on discharge breakdown, and $p_{1}$ increases accordingly.

When $P_{w}$ is 3-8 MPa, the small bubbles almost completely disappear, and the discharge breakdown is directly inhibited by $P_{w}$, leading to decreased shock wave energy. Therefore, the inhibitory effect of $P_{w}$ on the discharge breakdown is larger than the promotion effect on shock wave transfer at this stage, and $p_{1}$ decreases as $P_{w}$ increases.

Incident wave velocity $\left(D_{1}\right)$ at sensor 4 was obtained from transfer time $(t$-) distance $(x)$ curve. Figure 10 shows a typical curve for the case of $P_{w}=1 \mathrm{MPa}$ and $U_{m}=9 \mathrm{kV}$.

Shock wave velocities were approximately 1530 and $1520 \mathrm{~m} / \mathrm{s}$ at $1.5 \mathrm{~m}$ and $2.5 \mathrm{~m}$, respectively, from the electrode (sensors 1 and 4, resp.). For the shock wave transfer within $3 \mathrm{~m}$, a very slight attenuation of shock velocity was observed, and the curve is approximately a straight line.

Figure 11 shows $D_{1}$ at sensor 4 ( $2.5 \mathrm{~m}$ from the electrode) under different charging voltages $\left(U_{m}\right)$ and hydrostatic pressures $\left(P_{w}\right)$, as discussed above.

Incident wave velocity is mainly affected by $U_{m}$ and $P_{w}$. The charging voltage specifies the energy injected into the plasma channel, which is converted to mechanical energy, generating the perturbation and hence the shock wave. Increased perturbation energy produces increased $D_{1}$ following the Rankine-Hugoniot relations [23]. Previous research has established that $D_{1}$ increases with increasing hydrostatic pressure [24]. Figure 11 shows that $D_{1}$ increases with increasing $U_{m}$ for fixed $P_{w}$ which is consistent with the above theoretical analysis. 

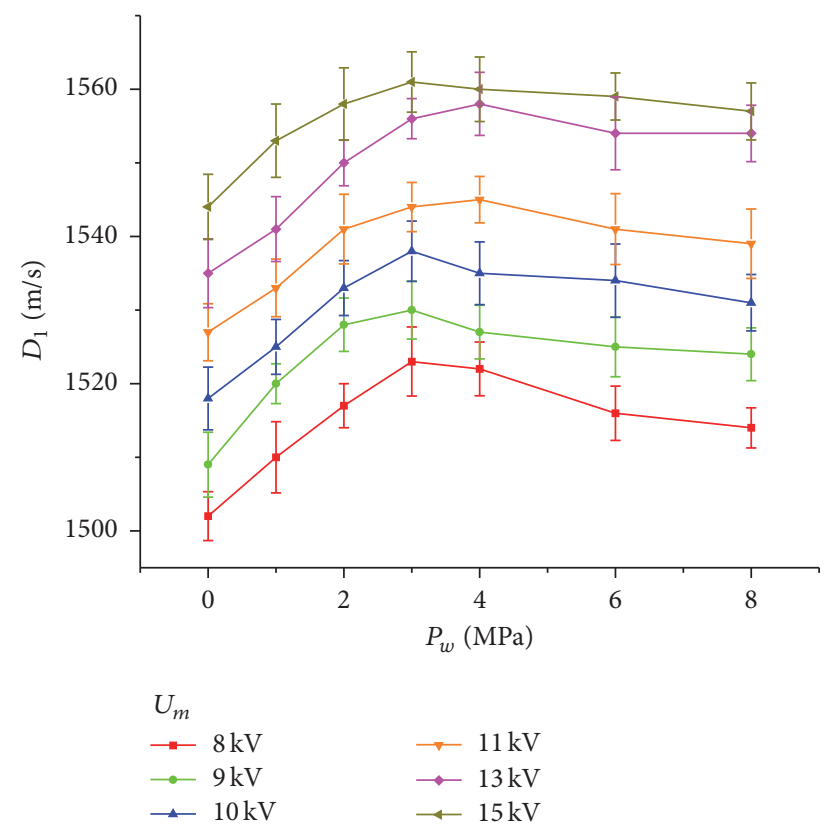

FIGURE 11: Incident shock wave velocity $\left(D_{1}\right)$ at $2.5 \mathrm{~m}$ from the electrode.

For fixed $U_{m}, D_{1}$ first increases then with increasing $P_{w}$, for $P_{w}=0-3 \mathrm{MPa}, D_{1}$ increases significantly. Then it shows a slow decrease for $P_{w}=3-8 \mathrm{MPa}$. The main reasons for this is the inhibitory effect of $P_{w}$ on the discharge breakdown and the promotional effect on $p_{1}$. When $P_{w}=0-3 \mathrm{MPa}$, original small bubbles remain in the water, weakening the inhibitory effect of $P_{w}$ on discharge breakdown. Since the perturbation of water from the discharge breakdown is not significantly negative affected by the low hydrostatic pressure, the promotion of $D_{1}$ from increased $P_{w}$ shows significantly.

However, the bubbles gradually disappear as $P_{w}=3-$ $8 \mathrm{MPa}$, hence the breakdown field strength of water medium increases, and the discharge breakdown is inhibited with increased $P_{w}$. The perturbation energy reduces, and $D_{1}$ decreases. However, the promotion effect of $P_{w}$ on $D_{1}$ is less significant than its inhibitory effect on the discharge breakdown, and $D_{1}$ decreases slowly as $P_{w}$ increases 3-8 MPa.

4.2. Characteristics of the Reflection Shock Wave. In the second part of the experiment (as shown in Figure 6), the shock wave collides with the rigid wall at $2.5 \mathrm{~m}$ from the electrode, forming a normal reflection shock wave. With respect to the incident wave, there is a large change in the peak pressure $\left(p_{2}\right)$ and velocity $\left(D_{2}\right)$ of the reflection shock wave. The shock wave peak pressure reflection coefficient also shows significant changes for various hydrostatic pressures and charging voltages. This has direct significance to reflection characteristics of shock waves. Figure 12 shows $p_{2}$ for different $P_{w}$ and $U_{m}$.

The peak pressure $\left(p_{2}\right)$ of the reflection shock wave is significantly higher than $p_{1}$ of the corresponding incident shock wave (compare Figures 12 and 9). For fixed $P_{w}, p_{2}$

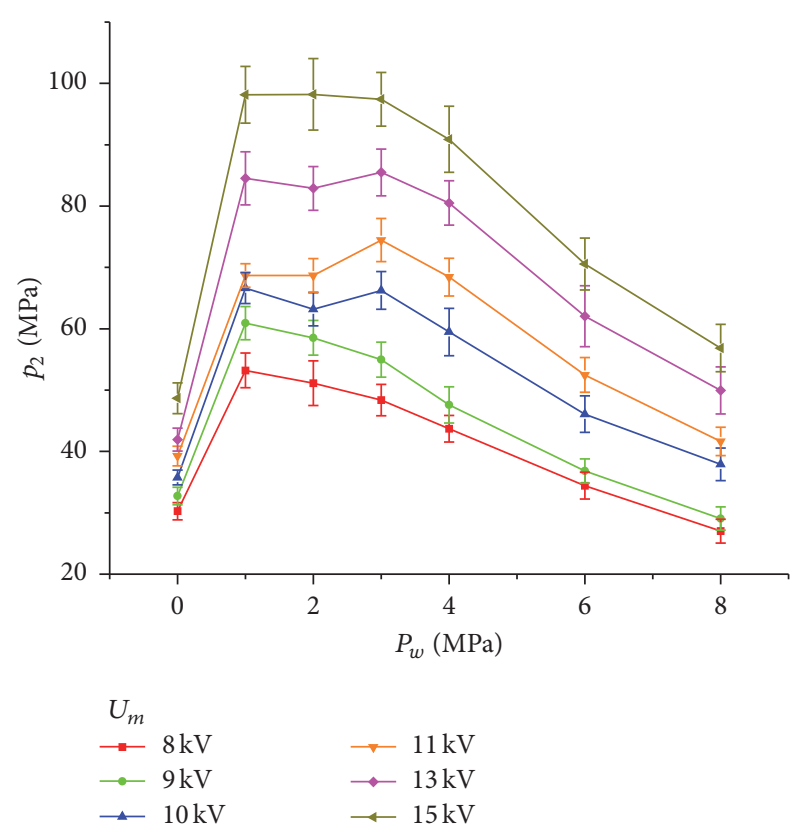

FIGURE 12: Peak pressure $\left(p_{2}\right)$ of the reflection shock wave measured at $2.5 \mathrm{~m}$ from electrode.

increases with increasing $U_{m}$. The energy in the plasma channel increases with increasing charging voltage, increasing $p_{1}$. The incident shock wave generates a large perturbation upon reflection, and the physical variables (pressure, density, and particle velocity) at the position all change suddenly. Increased $p_{1}$ leads to larger changes of the physical variables at reflection. The state behind the incident shock wave is the physical state of the reflection shock front medium, and the reflection shock wave forms the superposition on this high energy state [25]. Due to the superposition of shock wave, $p_{2}$ is significantly higher than $p_{1}$.

However, the above analysis does not consider the influence of $P_{w}$ on the reflection shock wave. For a given $U_{m}$, different $P_{w}$ cause differences in the discharge breakdown and $p_{1}$. Moreover, the reflection pressure $\left(p_{2}\right)$ is directly affected by incidence pressure $\left(p_{1}\right)$, and they have certain relationship under different $P_{w}$ conditions. Therefore, the influence of $P_{w}$ on the reflection shock wave needs to be determined. We used the peak pressure reflection coefficient, $n=p_{2} / p_{1}$ [26]. To investigate this effect, the reflection coefficient $(n)$ can accurately reflect the reflection characteristics of the peak pressure of shock wave under different hydrostatic pressures.

The theoretical value of $n$ can be calculated from (5), and the actual value can be obtained from experimental data. All the parameters of (5) can be accurately measured before reflection of the shock wave. The coefficient $\gamma$ can be determined from $c_{0}$ and $D_{1}$, for different $P_{w}$ and $U_{m}$ from (10), as shown in Figure 13.

The coefficient values $(\gamma)$ are in the range 3-3.14. The $\gamma$ has the specific approximate values, which have small fluctuation under the effects of different hydrostatic pressures and charging voltages. And it is helpful to accurately calculate the theoretical values of reflection coefficient $(n)$. 


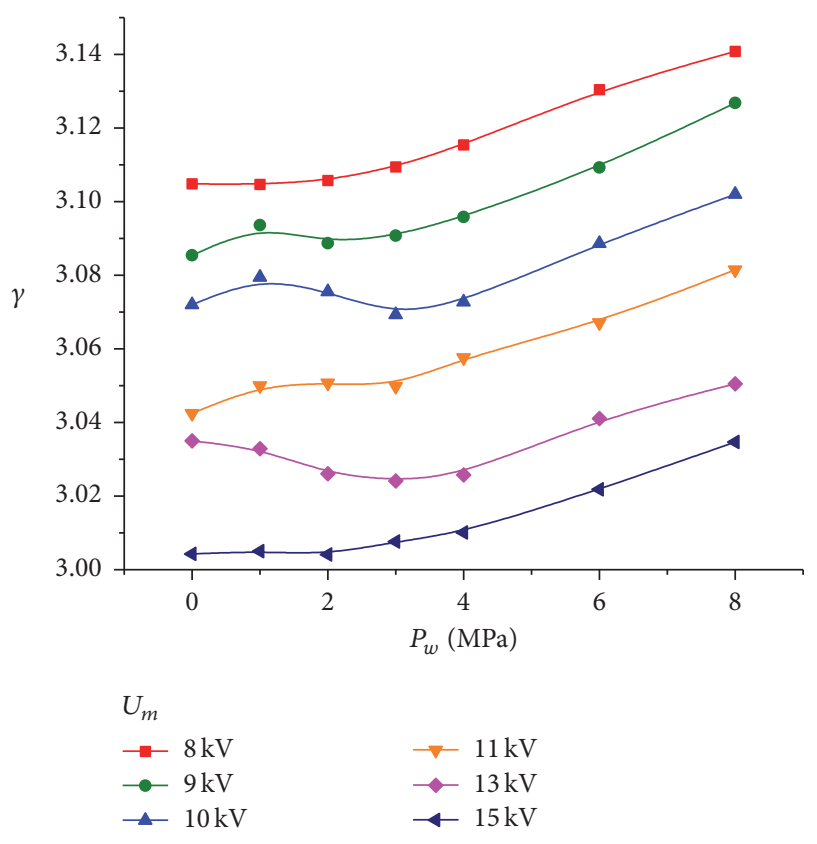

Figure 13: Values of coefficient $\gamma$ for different hydrostatic pressures and charging voltages.

The calculated $\gamma$ (Figure 13) and measured $p_{1}$ (Figure 9) were substituted into (5) to obtain the theoretical calculation values for $n$. The experimental values of $n$ were the direct ratio of measured $p_{2}$ (Figure 12) and $p_{1}$ (Figure 9). The dotted and solid lines denote the theoretical calculation values and experimental values in Figure 14, respectively.

The reflection coefficient $(n)$ is the ratio of peak pressure of the reflection shock wave to the peak pressure of incident shock wave. It can be seen from Figure 14 that theoretical and experimental $n$ decrease with increasing $P_{w}$ for fixed $U_{m}$. Thus, $P_{w}$ has a negative effect on $p_{2}$ and significantly inhibits superposition in the reflection process.

The mechanism can be described as below, the shock wave has obvious characteristics of a longitudinal wave, and when a molecule shifts horizontally into the rigid wall, another molecule around the rigid wall will reversely shift horizontally away. Pressure is the force per unit area, and force is the variation of medium momentum per unit time $(F=d I / d t$; where $F$ is force, $I$ is momentum, and $t$ is time) [27]. From a microcosmic perspective, the medium here means water molecules. For the molecule bounced back from the wall after the elastic collision, momentum is in the opposite direction to the incident wave, and the amplitude of reflection momentum superposes on the amplitude of incidence momentum. The state of reflection wave front (i.e., the state behind the incidence wave) is disturbed to varying degrees due to $P_{w}$ and $p_{1}$, causing significant changes to the velocity of corresponding reflection particles, with corresponding changes to the reflection momentum amplitude. The peak pressure of the reflection wave is caused by superposition of the incident and reflection shock wave momentum. Since high $P_{w}$ inhibits perturbation of the reflection wave front, the perturbation weakens, and reflection particle velocities are reduced, with corresponding reduction in reflection momentum. Thus, the superposition of incidence and reflection momentum decreases, and $n$ decreases.

Theoretical and experimental $n$ values increase with increasing $U_{m}$ for fixed $P_{w}$. The mechanism is similar to that discussed above. Increased $U_{m}$ provides increased energy for the shock wave and larger perturbation behind the incidence wave. Therefore, the particle velocity of the reflection wave at this perturbation state also increases relatively; the reflection momentum value also increases, with increased superposition of incidence and reflection momentum. Thus, the corresponding $p_{2}$ increases, and $n$ also increases.

Experimental values of $n$ are all lower than the corresponding theoretical values for a given $P_{w}$. The reasons for this are the following.

(1) The theoretical analysis considers the surface of the rigid wall to be smooth and all individual molecular collisions to be elastic and normal to the surface [28]. However, practically, the wall surface is rough, and some reflections are inevitably nonnormal to the nominal flat surface, leading to incomplete superposition of momentum. Consequently, the vector components of partial reflection momenta are lost.

(2) Sensor 4 is very close to the rigid wall (within $5 \mathrm{~mm}$ ). However, given the thicknesses of the incidence and reflection waves [29], the thickness of the superposition area of the reflected shock wave is much smaller than the distance to sensor 4 . Thus, the measured $p_{2}$ includes some small attenuation. Above stated are the main reasons for the smaller experimental values.

(3) The deviation between experimental and theoretical $n$ $0 \mathrm{MPa}$ is significantly larger than for other $P_{w}$. This is due to the difficulty of ensuring the tube is completely full of water under nominal zero pressure, since the presence of even a small amount of air or a few bubbles has a negative effect on shock wave reflection. This situation is equivalent to the propagation of shock wave in discontinuous medium [30].

The reflection wave velocity can be calculated by the transfer time versus propagation distance curve, similar to the incident shock wave. For example, Figure 15 shows the transfer time ( $t$-) distance $(x)$ curve of the shock wave for $P_{w}=1 \mathrm{MPa}$ and $U_{m}=9 \mathrm{kV}$, where the rigid wall is shown at $2.5 \mathrm{~m}$, and the red and blue lines denote the incidence and reflection wave, respectively.

The approximate velocity was calculated from Figure 15 as 1520 and $1480 \mathrm{~m} / \mathrm{s}$ for the incident and reflection shock waves, respectively. The reflection shock wave velocity is significantly lower than that of the incident shock wave because some shock wave energy is lost in the collision with the rigid wall [31]. Moreover, after the reflection superposition process, the superposition effect disappears for the state behind the reflection wave, and the perturbation reverts to a constant value. This is equivalent to a negative pressure state behind the reflection wave. The rarefaction wave formed during this instantaneous negative pressure state also reduces the energy of the reflection wave. Consequently the reflection 

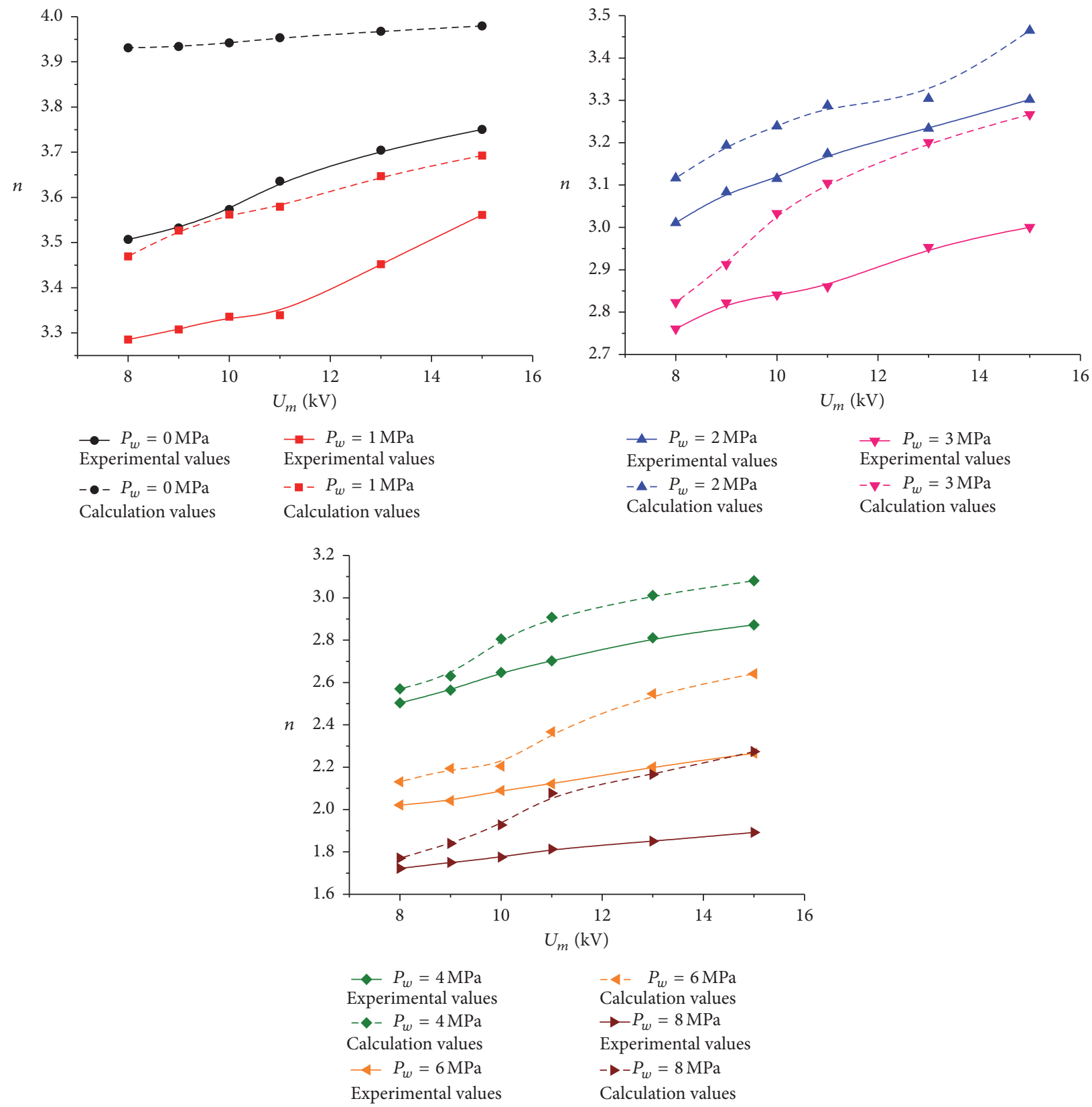

FIGURE 14: Theoretical calculation and experimental values for the reflection coefficient $(n)$.

wave velocity is lower than the velocity of the incidence wave. The results are consistent with the other states.

The reflection wave velocity at $2.5 \mathrm{~m}$ from the electrode for different $U_{m}$ and $P_{w}$ was as shown in Figure 16.

It can be seen from Figure 16 that reflection wave velocity $\left(D_{2}\right)$ increases with increasing $P_{w}$ for fixed $U_{m}$. This is because the propagation velocity of a wave in the medium increases with increasing $P_{w}$. And $D_{2}$ steadily increases with increasing $U_{m}$ for fixed $P_{w}$, the mechanism can be briefly described as below: the higher the velocity of incident wave, the higher the velocity of the reflection wave.

Comparing $D_{2}$ and $D_{1}$ (Figures 16 and 11, resp.), $D_{2}$ are relatively steady for $U_{m}=8-15 \mathrm{kV}$ at constant $P_{w}$, whereas there are large differences in $D_{1}$. Thus, the effect of $U_{m}$ on $D_{2}$ is much less than on $D_{1}$. The charging voltage is the main factor influencing $D_{1}$ before reflection. After reflection, the perturbation energy of the wave source is a secondary influencing factor for $D_{2}$, in terms of relativity to $D_{1}$. As $P_{w}$ increases, $D_{2}$ increases smoothly, which is similar to the general characteristics of sound waves in water [32]. Thus, the reflection shock wave is little affected by the wave source, and $D_{2}$ is close to the sound velocity at corresponding $P_{w}$. The velocity of sound is $1447,1451,1455,1459,1463,1471$, and $1479 \mathrm{~m} / \mathrm{s}$ at $10^{\circ} \mathrm{C}$ when the hydrostatic pressure is $0,1,2,3,4,6$, and $8 \mathrm{MPa}$, respectively. Therefore, the reflection wave can be considered a weak shock wave [33]. The velocity of reflection 


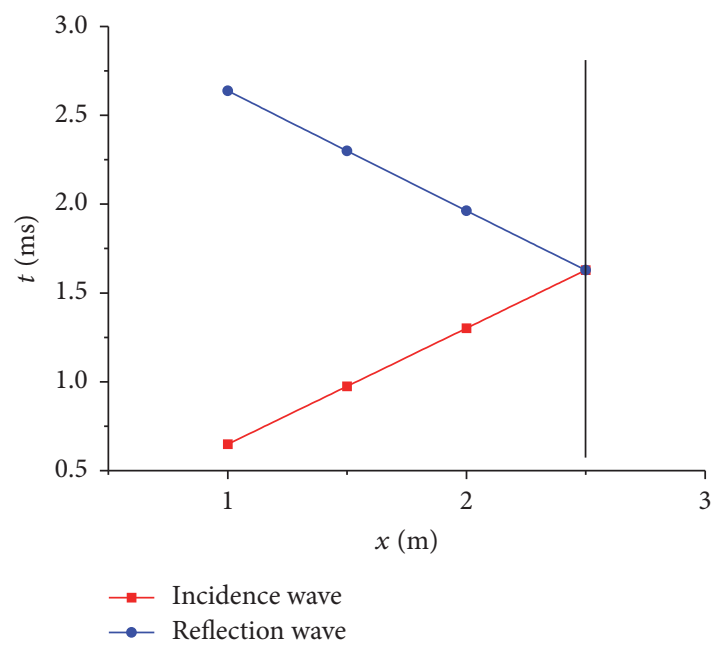

FIGURE 15: Transfer time $(t-)$ distance $(x)$ curve of the shock wave for $P_{w}=1 \mathrm{MPa}$ and $U_{m}=9 \mathrm{kV}$.

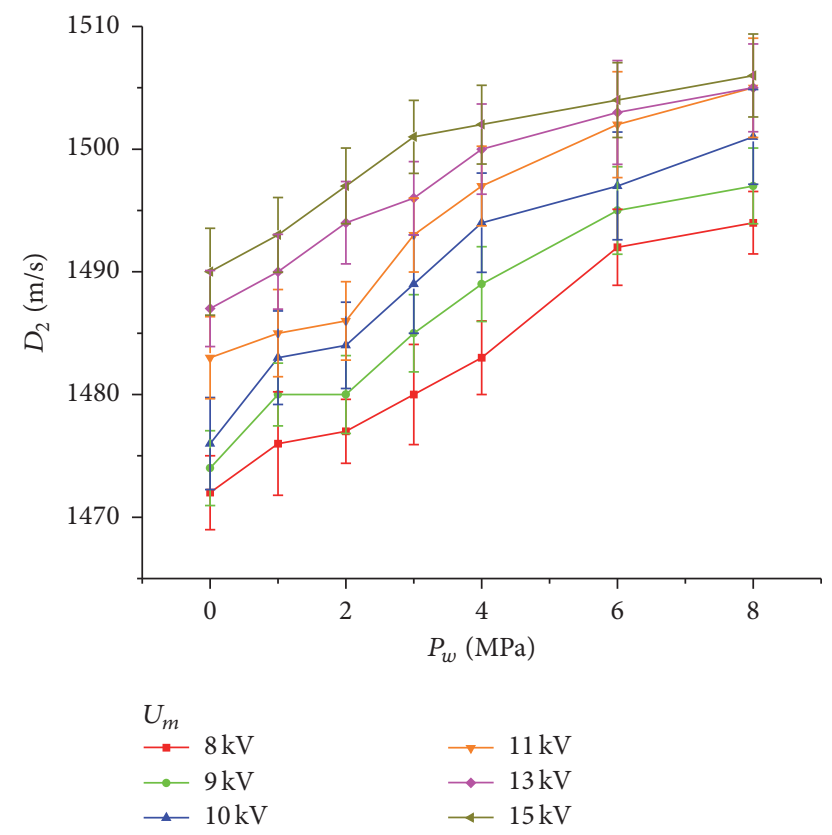

FIGURE 16: Reflection shock wave velocity $\left(D_{2}\right)$ at $2.5 \mathrm{~m}$ from the electrode.

wave is closer to the velocity of sound for higher hydrostatic pressure; that is, the weak shock wave characteristics of the reflection wave become more pronounced for increasing $P_{w}$.

\section{Conclusions}

(1) The pressure and velocity of the incident shock wave $2.5 \mathrm{~m}$ from the electrode both increase when hydrostatic pressure $P_{w}=0-3 \mathrm{MPa}$ due to the inhibitory effect of $P_{w}$ on discharge breakdown and the promotion effect on shock wave transfer. However, the pressure and velocity of the incident shock wave gradually decreased for $P_{w}=3-8 \mathrm{MPa}$.
(2) The peak pressure of the normal reflection shock wave on the rigid wall was significantly higher than that of the incident shock wave. The state behind the incident shock wave was the physical state of the reflection shock front. Thus, the reflection shock wave was superposed onto this high energy state. The peak pressure of the incident wave increased with increasing charging voltage $\left(U_{m}\right)$. Since the reflection wave was superposed on the incident shock wave, the peak pressure of reflection shock wave was also higher, which was consistent with the RankineHugoniot relations on the discontinuity surface of the shock wave.

(3) The influence of $P_{w}$ on the reflection shock wave was determined by the peak pressure reflection coefficient, $n$. Theoretical calculation and experimental $n$ decreased with increasing $P_{w}$. Therefore, $P_{w}$ had a negative effect on the peak pressure of reflection shock wave, $p_{2}$, which significantly inhibited pressure superposition in the reflection process.

(4) For fixed $P_{w}$, reflection wave velocities $\left(D_{2}\right)$ corresponding to different $U_{m}$ were close. However, there were large differences between the corresponding incidence wave velocities. The effect of $U_{m}$ on $D_{2}$ is much smaller than that on the velocity of incidence wave $\left(D_{1}\right)$. While $U_{m}$ was the main factor influencing $D_{1}$, after reflection from the rigid wall, $D_{2}$ was little affected by the perturbation energy of the wave source (i.e., $U_{m}$ ), and it belonged to a secondary influencing factor.

(5) The experiments showed the reflection shock wave could be considered a weak shock wave after collision of the incident shock wave with the rigid wall. Higher $P_{w}$ produced $D_{2}$ closer to the velocity of sound at the corresponding $P_{w}$ and made the weak shock wave characteristics more obvious for the reflection shock wave.

\section{Competing Interests}

The authors declare that there is no conflict of interests regarding the publication of this paper.

\section{Acknowledgments}

This work is supported by the Shanxi Coalbed Methane Foundation of China (2012012012).

\section{References}

[1] J. S. Zhu, X. M. Hu, P. Wang, and J. Chen, "A review on research progress in explosion mechanics and impact dynamics," Advances in Mechanics, vol. 40, no. 4, pp. 400-423, 2010.

[2] Z. Y. Qin, G. N. Zuo, and Y. R. Wang, High-Voltage Strong-Pulse Discharge and Its Applications, Beijing University of Technology Press, Beijing, China, 2000. 
[3] I. Z. OKun, "Plasma parameters in a pulsed discharge in a liquid," Soviet Physics: Technical Physics, vol. 16, no. 2, pp. 227231, 1971.

[4] R. Courant and K. O. Friedrichs, Supersonic Flow and Shock Waves, Beijing World Publishing Corporation, Beijing, China, 2006.

[5] X. M. Yang, Numerical Simulation for Explosion and Phenomena, University of Science and Technology of China Press, Hefei, China, 2010.

[6] W. X. Li, One-Dimensional Nonsteady Flow and Shock Waves, National Defence Industry Press, Beijing, China, 2003.

[7] S. P. Marsh, LASL Shock Hugoniot Data, University of California Press, Berkeley, Calif, USA, 1980.

[8] Q. Zhao, S. Z. Liu, and H. H. Tong, Plasma Technology and Its Applications, National Defence Industry Press, Beijing, China, 2009.

[9] J. S. Chang, K. Urashima, and Y. Uchida, "Characteristics of Pulsed Arc Electrohydraulic Discharges and Their Application to Water Treatment," Research Reports of the Faculty of Engineering, Tokyo Denki University, vol. 50, p. 1, 2002.

[10] J. M. Yang, Z. F. Li, Y. J. Zhu, and Z. G. Zhai, "Shock wave propagation and interactions," Advances in Mechanics, vol. 46, no. 1, pp. 541-587, 2016.

[11] G. Touya, T. Reess, L. Pécastaing, A. Gibert, and P. Domens, "Development of subsonic electrical discharges in water and measurements of the associated pressure waves," Journal of Physics D: Applied Physics, vol. 39, no. 24, article 021, pp. 52365244, 2006.

[12] B.-M. Bian, L. Yang, Z.-H. Li, X. Chen, A.-Z. He, and Z.-H. Shen, "The study of self-simulating properties of spherical shock front attenuation," Acta Physica Sinica, vol. 53, no. 3, pp. 840-843, 2004.

[13] D. Yan, D. Bian, J. Zhao, Z. Yin, S. Jia, and J. Feng, "Efficiency analysis of bubble pulsation formed by high-voltage discharge in nonfree field water," Advances in Mechanical Engineering, vol. 8, no. 8, pp. 1-11, 2016.

[14] Z. H. Zeng, Research of Large Pulsed Current Test, Nanjing University of Science and Technology, Nanjing, China, 2006.

[15] Y. S. Liu, "Method of using P6015A detection head to measure DC high voltage," Electrotechnics Electric, vol. 4, no. 1, pp. 42-44, 2006.

[16] X. P. Lu, Y. Pan, and H. H. Zhang, "The electrical and acoustical characteristics of pulsed discharge in water," Acta Physica Sinica, vol. 51, no. 7, pp. 1549-1553, 2002.

[17] B. Sun, Discharge Plasma in Liquid and Its Applications, Science Press, Beijing, China, 2013.

[18] D. Yan, D. Bian, J. Zhao, and S. Niu, "Study of the electrical characteristics, shock-wave pressure characteristics, and attenuation law based on pulse discharge in water," Shock and Vibration, vol. 2016, Article ID 6412309, 11 pages, 2016.

[19] W. Jia, A. C. Qiu, and F. J. Sun, "Effects of the pressure under the several hundred nanosecond pulse on the breakdown characteristics of the water switch," High Voltage Engineering, vol. 32, no. 1, pp. 50-52, 2006.

[20] K. C. Kao, "Breakdown of dielectric liquids," in Proceedings of the AIEE Winter Meeting, pp. 60-84, 1960.

[21] E. V. Yanshin, I. T. Ovchinnikov, and Y. N. Vershinin, "Optical study of manosecond prebreakdown phenomena in water," Soviet Physics: Technical Physics, vol. 18, pp. 1303-1306, 1974.

[22] V. Y. Ushakov, Impulse Breakdown of Liquids, Springer, Berlin, Germany, 2007.
[23] P. O. K. Krehl, "The classical Rankine-Hugoniot jump conditions, an important cornerstone of modern shock wave physics: ideal assumptions vs. reality," European Physical Journal H, vol. 40, no. 2, pp. 159-204, 2015.

[24] A. Saul and W. Wagner, "A fundamental equation for water covering the range from the melting line to $1273 \mathrm{~K}$ at pressures up to $25000 \mathrm{MPa}$," Journal of Physical and Chemical Reference Data, vol. 18, no. 4, pp. 1537-1564, 1989.

[25] S. C. Frank, Waves Berkeley Physics Course, vol. 3, China Machine Press, Beijing, China, 2014.

[26] H. J. Pain, The Physics of Vibrations and Waves, People's Education Press, Beijing, China, 1980.

[27] W. D. Shi, "Momentum theorem and its inferences in fluid mechanics," Journal of Jiangsu University, vol. 12, no. 1, pp. 6370, 1991.

[28] J. Fan, S. L. Peng, H. L. Liu, and Q. Shen, "Program marking of surface elements and the determination of molecular surface reflection in position element algorithm of DSMC method," Chinese Journal of Theoretical and Applied Mechanics, vol. 31, no. 6, pp. 671-676, 1999.

[29] C. Guo, Computation for the Thickness of Shock Wave, Inner Mongolia University, Hohhot, China, 2007.

[30] Y. Z. Feng, A First Course in Continuum Mechanics, Tsinghua University Press, Beijing, China, 2009.

[31] R.-C. Shi, R.-Y. Huang, G.-Y. Wang, Y.-K. Wang, and Y.-C. Li, "Numerical simulation of underwater shock wave propagation in the vicinity of rigid wall based on ghost fluid method," Shock and Vibration, vol. 2015, Article ID 467376, 16 pages, 2015.

[32] D. Z. Wang and E. C. Shang, Underwater Acoustics, Science Press, Beijing, China, 2016.

[33] W. H. Tang, Shock Wave Physics, Science Press, Beijing, China, 2011. 


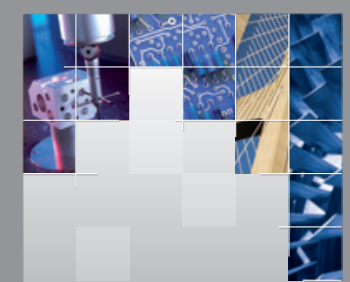

\section{Enfincering}
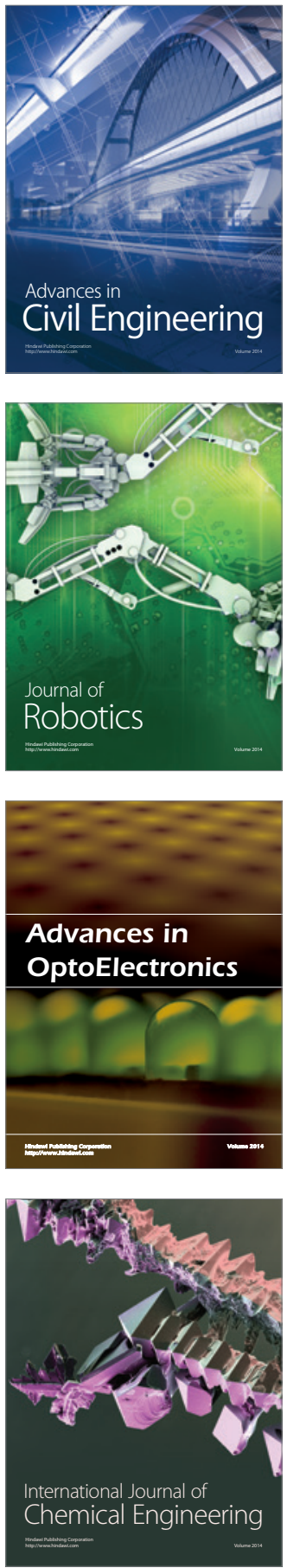

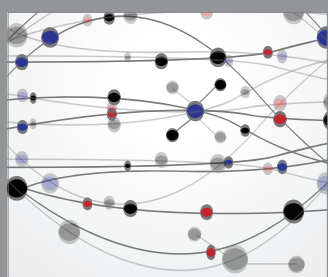

The Scientific World Journal

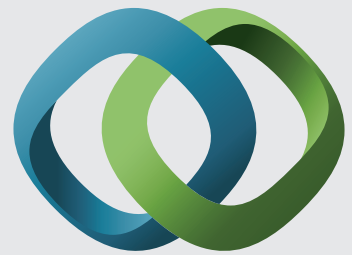

\section{Hindawi}

Submit your manuscripts at

https://www.hindawi.com
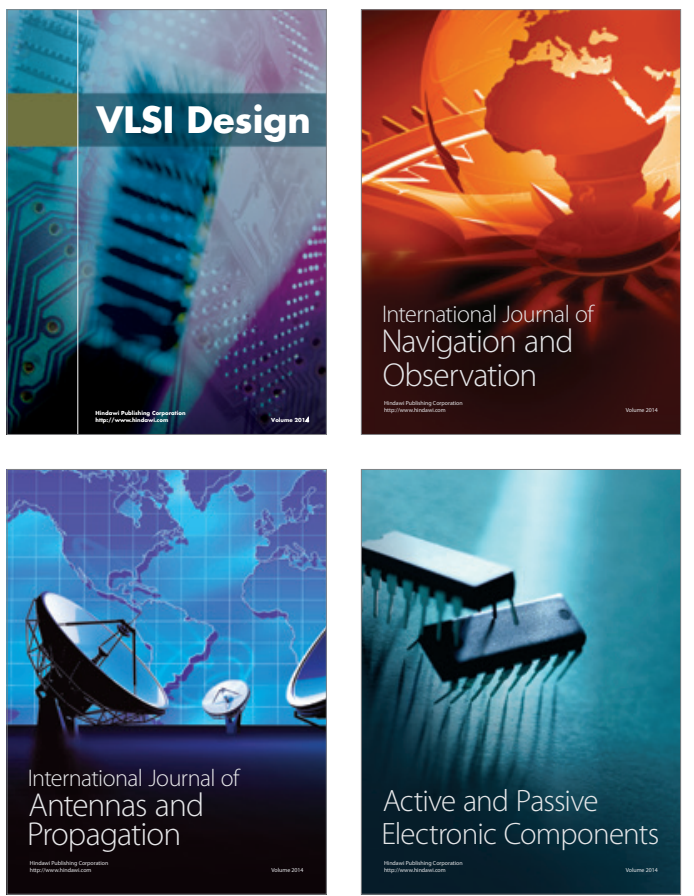
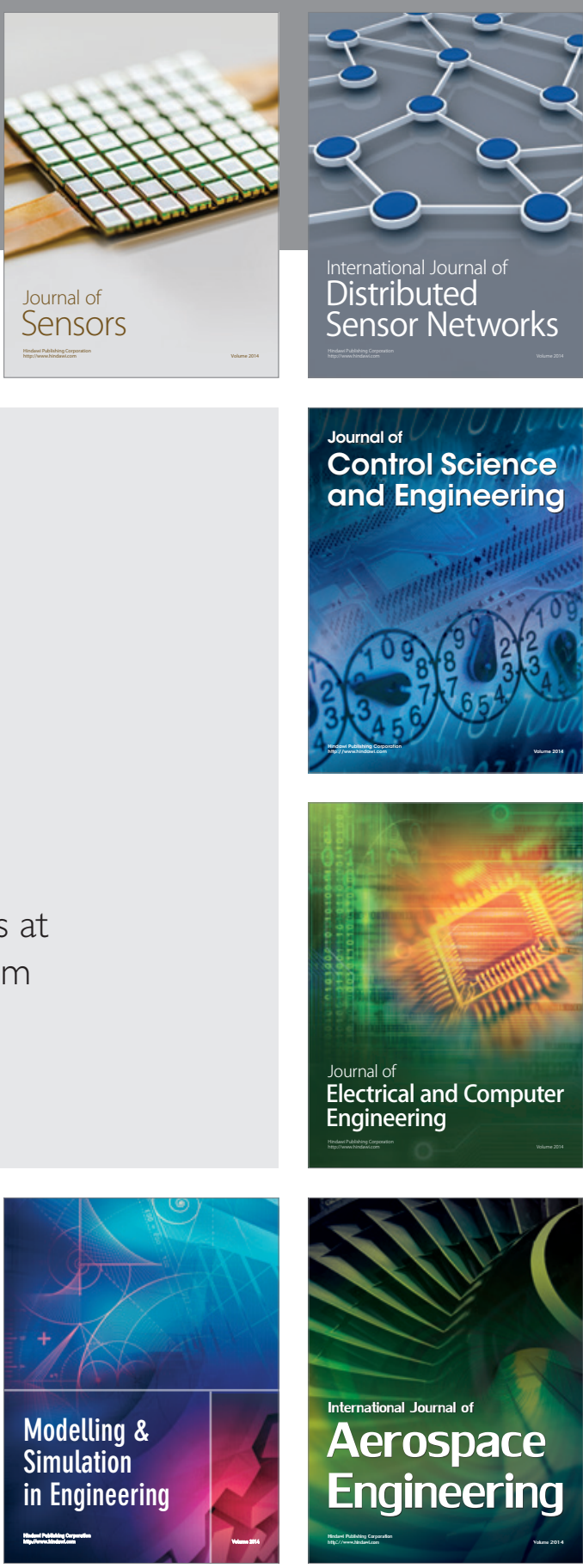

International Journal of

Distributed

Sensor Networks

$-$

Joumal of

Control Science

and Engineering
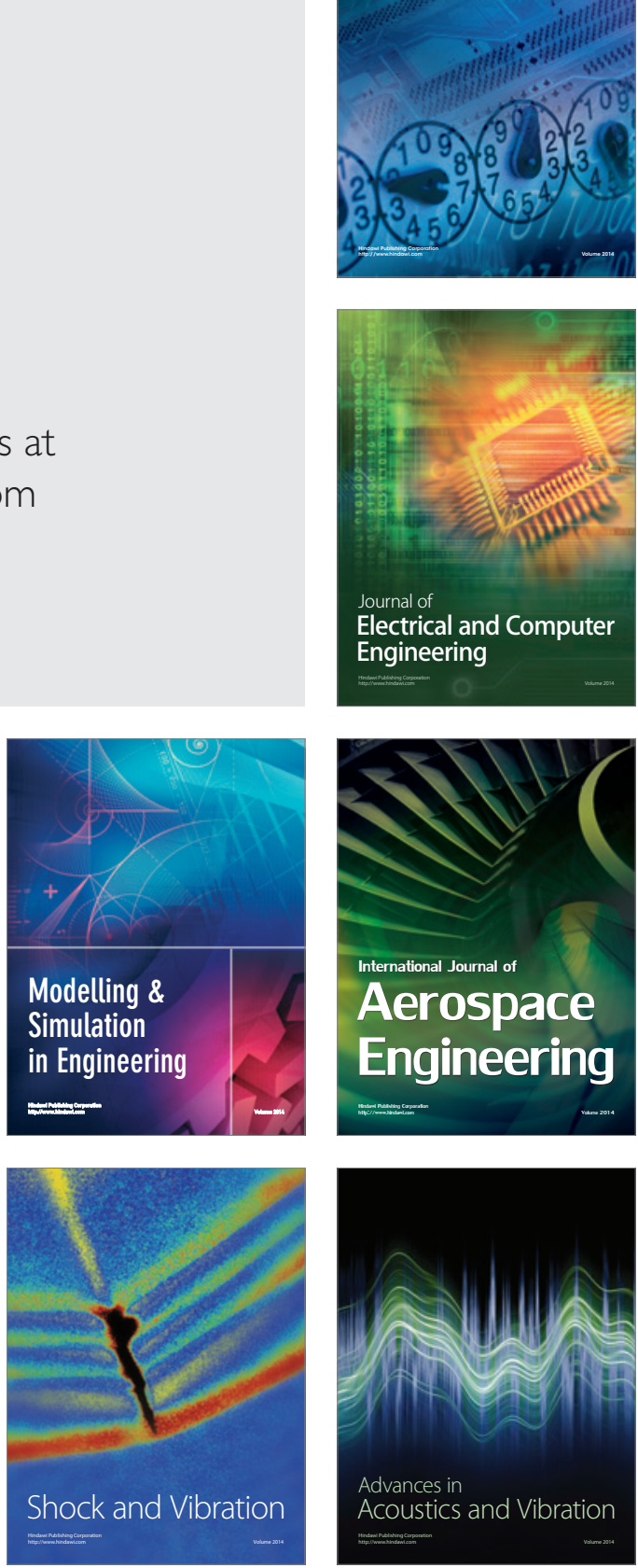